\title{
Isolation, Identification and Antibiotic Susceptibility Pattern of Urinary Tract Infection Bacterial Isolates
}

\author{
Ahmed Said ${ }^{1}$, Mamdouh S El-Gamal ${ }^{1}$, Mohammed Abu-Elghait ${ }^{1}$ (D), Salem S Salem 1,*(D) \\ 1 Botany and Microbiology Department, Faculty of Science, AL-Azhar University, Nasr City, Cairo-11884, Egypt \\ * Correspondence: salemsalahsalem@azhar.edu.eg (S.S.S.);
}

Scopus Author ID 57202162965

Received: 19.02.2021; Revised: 28.03.2021; Accepted: 5.04.2021; Published: 9.05.2021

\begin{abstract}
Recently, the world's problem has become in the spread of microbes and the extent of their impact on public health, especially the problem of urinary tract infections (UTIs). Therefore, the purpose of the study was to clarify the extent of infection of the urinary tract and the extent of development of these microbes in resistance to antibiotics (antibiotic susceptibility pattern). The pathogens are isolated from hospitals and private medical centers. In this study, two hundred twentyfive urine samples were collected from various sources, where 126 patients were infected with bacteria while 99 patients had no infection. In Gram-negative bacteria, the most common bacteria were E. coli (55.5\%) followed by Klebsiella spp (23.0\%), Proteus spp (7.14\%), Pseudomonas spp (6.34\%), and Acinetobacter spp (3.96\%). In the Gram-positive bacteria Staphylococcus spp (2.38\%) and Enterococcus spp (1.58\%) were found. Antibiotic susceptibilities for Gram-negative and positive bacteria were investigated according to clinical laboratory standard institute(CLSI 2019). In Gramnegative bacteria, Amikcin, Impinem, and levofloxacin were sensitive. However, in Gram-positive bacteria, levofloxacin, ofloxacin, and linezolid were sensitive. At the same time, most of the remaining antibiotics are $100 \%$ resistant to pathogenic-bacterial isolates. This study showed bacteria that are isolated from urine which causing urinary tract infection and showed resistance to almost all antibiotics, so UTI is difficult to treat by common antibiotics.
\end{abstract}

Keywords: urinary tract infections; bacterial pathogens; antibiotic susceptibility.

(C) 2021 by the authors. This article is an open-access article distributed under the terms and conditions of the Creative Commons Attribution (CC BY) license (https://creativecommons.org/licenses/by/4.0/).

\section{Introduction}

Urinary tract infection (UTIs) refers to the presence or growth of microorganisms in any part of the urinary tract and represents bacterial infection more common in humankind $[1,2]$. Urinary tract infections (UTIs) 150 million people are infected with this infection every year. It is one of the most common bacterial infections $[3,4]$. The cause of the urinary tract infection due to the proliferation of bacteria in the urinary tract. The clinical manifestations depend on the infected part of the urinary tract, organism type, infection rigidity, and ability of the patient's immune response [5,6]. Urinary tract infection includes a population of (newborn, mature girls, sexually active females, and elderly females) [7]. In pregnant women, about $8 \%$ have a UTI, and an untreated UTI can have harmful effects on them[8]. UTIs lead to major economic and public health burdens and affect the quality of life for affected individuals [9,10,11,12]. Escherichia coli is responsible for uncomplicated pyelonephritis and cystitis and is followed by other Enterobacteriaceae species such as Klebsiella pneumoniae and Proteus mirabilis and by Gram-positive bacteria such as Staphylococcus saprophyticus, and 
Enterococcus faecalis $[4,13]$. Various agents are used to treating uncomplicated UTIs and pyelonephritis by international guidelines such as trimethoprim-sulfamethoxazole, fosfomycin trometamol, pivmecillinam, nitrofurantoin monohydrate, fluoroquinolones, and beta-lactams [14]. The development of bacterial resistance to presently available antibiotics results from the indiscriminate and widespread use of antibiotics [15,16]. Bacteria producing extendedspectrum beta-lactamases (ESBLs), showing resistance to most antibiotics except for the carbapenem group, are steadily increasing in the population $[17,18]$.

Furthermore, the targeting bacteria may become resistant to effective antibiotics and become unable to kill bacterial cells. This produces antibiotic-resistant strains of E. coli [18]. Multiple antibiotics resistance pathogens result from the use of antibiotics more frequently for those individuals $[19,20]$. Therefore, the purpose of the study was to isolate and identify the bacteria that cause UTI and their development in antibiotic resistance (antibiotic susceptibility pattern).

\section{Materials and Methods.}

\subsection{Specimen localities.}

All samples were collected from different places, including some hospitals and Egyptian laboratories, and the count of specimens 225. Samples were collected in sterile cups, kept in an icebox, and transferred to the bacteriological laboratory at once.

\subsection{Isolation media.}

Five types of different enrichment and selective media were used for the isolation and identification of bacteria.

2.2.1. Cystine-lactose-electrolyte-deficient medium (CLED) (g/L).

CLED Agar (Cystine-Lactose-Electrolyte-Deficient Agar) is a differential culture medium for isolating and enumerating bacteria from urine. It supports the growth of urinary pathogens and contaminants. However, it prevents undue swarming of Proteus species due to its lack of electrolytes. Its composition was as follows: Beef extract $3.0 \mathrm{~g}$, Pancreatic digest of casein $4.0 \mathrm{~g}$, Lactose $10.0 \mathrm{~g}$, Bromthymol blue $0.02 \mathrm{~g}$, L-cystin 128.0 $\mathrm{mg}$, and agar $15.0 \mathrm{~g}$. The $\mathrm{pH}$ was adjusted to 6.8 .

\subsubsection{MacConkey agar (g/L).}

MacConkey agar was utilized to isolate and differentiate gram-negative enteric bacteria, coliform bacteria, intestinal bacterial pathogens, and bacteria from urine samples. And through the fermentation of lactose, it is possible to differentiate between gram-negative bacteria, and its composition was as follows: Lactose $10.0 \mathrm{~g}$, Bile salts $1.5 \mathrm{~g}, \mathrm{NaCl} 5.0 \mathrm{~g}$, Neutral red $0.05 \mathrm{~g}$, Crystal violet $0.001 \mathrm{~g}$, and agar $15.0 \mathrm{~g}$. The $\mathrm{pH}$ was adjusted to 7.4.

\subsubsection{Manitol salt agar (g/L).}

It was used for the selective isolation and differentiation of Staphylococcus aureus, and its composition was as follows: D-Mannitol $10.0 \mathrm{~g}, \mathrm{NaCl} 75.0 \mathrm{~g}$, Beef extract $1.0 \mathrm{~g}$, Phenol red $0.025 \mathrm{~g}$, and agar $15.0 \mathrm{~g}$. The $\mathrm{pH}$ was adjusted to 7.4. 


\subsubsection{Bile aesculin agar $(\mathrm{g} / \mathrm{L})$.}

Bile aesculin agar was recommended for isolation and identification of group D Streptococci and Enterococci spp. Its composition was as follows: bile salts $15.0 \mathrm{~g}$, Pepton $14.0 \mathrm{~g}$, ferric citrate $0.5 \mathrm{~g}$, Aesculin $1.0 \mathrm{~g}$, and agar14.0 g. The $\mathrm{pH}$ was adjusted to 7.1.

\subsubsection{Cellobiose tryptophan Iron agar medium (CTIA-Tube) (g/L) [21]:}

CTIA medium was used to identify Gram-negative bacilli bacteria isolated from urine cultures, and its composition was as follows: Tryptone $20.0 \mathrm{~g}$, Yeast extract $3.0 \mathrm{~g}$, LTryptophan $5.0 \mathrm{~g}$, Sodium thiosulphate $0.3 \mathrm{~g}$, Ferric ammonium citrate $0.3 \mathrm{~g}$, Phenol red 0.003 g, Glucose $1.0 \mathrm{~g}$, Cellobiose $10.0 \mathrm{~g}, \mathrm{NaCl} 5.0 \mathrm{~g}$, and agar $17.0 \mathrm{~g}$. The $\mathrm{pH}$ was adjusted to 7.6.

\subsection{Assessment of bacterial population and purification of isolates}

The samples were cultured in nutrient agar media, CLED agar, and MacConkey agar and were incubated at $37^{\circ} \mathrm{C}$ for 24 hours. After the bacterial growth, the colonies' purity is checked, and then pick up the different colonies and kept in glycerol Broth media. Purity is checked out by colony morphology, Gram stain, and biochemical reaction, which are used to identify Gram-positive and Gram-negative bacteria [22].

\subsection{Identification of bacteria.}

\subsubsection{Identification of Gram-negative bacilli.}

According to the Manual of Methods for General Bacteriology, the morphological characteristics of the bacterial isolates have been studied on a scientific basis [23]. Gram stain was used as the first step to define according to the microorganisms' shape according to the Modification of gram stain. The isolates were inoculation on Macconkey agar(Oxoid) to differentiate between lactose fermentation and non-lactose fermentation incubated at $37^{\circ} \mathrm{C}$ after incubation, the isolates were inoculated on (CTIA-Tube) for $20-24 \mathrm{~h}$ at $35-37{ }^{\circ} \mathrm{C}$ [21]. The tube is unique for testing 5 biochemical characters: Glucose and Cellobiose fermentation, $\mathrm{H}_{2} \mathrm{~S}$ production, Tryptophan deaminase activity, and finally, indole production. The pseudomonas spp are characterized than Acinetobacter spp by oxidase test by method of Kovacs [24]. The Kovacs reagent consists of p-amino-dimethyl- alanine oxalate $(1 \%, \mathrm{w} / \mathrm{v}$, in distilled water). The test was performed after incubation bacterial isolates on (CTIA.Tube Agar) for $24 \mathrm{~h}$ at $37^{\circ} \mathrm{C}$ and flooding the surface with the reagent. The positive results give violet color.

\subsubsection{Identification of Gram-positive cocci.}

The bacteria inoculated on CLED media agar after identified by gram stain and inoculated on Mannitol salt media agar to allow the growth of pathogenic Staphylococcus bacteria and inoculated on Bile aesculin agar to identify some bacteria through of morphology of bacteria. The use of biochemical tests includes catalase test [24]. This test was characterized by determining the ability of the microbe to produce the catalase enzyme, as it breaks up Hydrogen peroxide into water and oxygen, so it appears as a gas bubble as an indicator of the exit of the oxygen gas and is done by adding a few drops of hydrogen peroxidase to a culture grown on nutrient agar. The tube coagulase test $[25,26]$ was used to determine pathogenic and non-pathogenic Staphylococcus spp. The coagulase test is positive when tube clotting occurs. 
It is done as follows: emulsify representative colony/colonies of the test organism in the plasma; incubate at $35-37^{\circ} \mathrm{C}$ and examine hourly up to $4 \mathrm{hr}$; do not shake or agitate the tube; gently slant and examine for a clot which gels the whole contents of the tube or forms a loose web of fibrin. Finally, all resistant microorganisms were confirmed by full biochemical tests by BIOMERIEUX VITEK2 SYSTEM.

\subsection{Antimicrobial susceptibility.}

Antimicrobial susceptibility testing was done using the disk diffusion method under Clinical and Laboratory Standards Institute [27] guidelines. One colony of the organism was emulsified in $1 \mathrm{ml}$ of sterile normal saline and mixed well; the turbidity was compared to 0.5 McFarland standards. A sterile cotton swab was used to inoculate the sample into MuellerHinton agar plates (Oxoid, Cambridge, UK). According to clinical and laboratory standard institute (CLSI), this media was used for antimicrobial susceptibility. It is low in sulfonamide, trimethoprim, and tetracycline inhibitors and provides satisfactory growth of most nonfastidious pathogens [28]. This media was prepared by dissolving the solid ingredients in one liter of distilled water (g/l): 17.5 Acid hydrolysate casein, 2.0 Beef extract, 1.5 Starch, and 17 Agar, $\mathrm{pH}$ adjusted to $7.3 \pm 0.1$ and autoclaving at $121^{\circ} \mathrm{C}$ for $15 \mathrm{mins}$ for sterilization. The used antibiotics are divided according to type of microorganisms where use the family of Enterobacteriaceae (Amikacin $(30 \mu \mathrm{g})$, Levofloxacin $(30 \mu \mathrm{g})$, Gentamycin (10 $\mu \mathrm{g})$, Impinem $(10 \mu \mathrm{g})$, Ofloxacin $(30 \mu \mathrm{g})$, Gatifloxacin $(30 \mu \mathrm{g})$, Naladixic acid $(30 \mu \mathrm{g})$, Streptomycin $(30 \mu \mathrm{g})$,

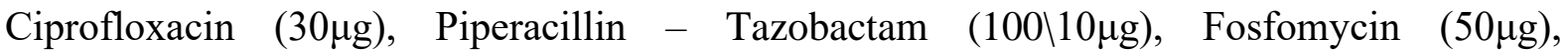
Nitrofurantoin $(300 \mu \mathrm{g})$, Cefotaxim $(30 \mu \mathrm{g})$, Cefepim $(30 \mu \mathrm{g})$, Cefoxitin $(30 \mu \mathrm{g})$, Ampicillin $(30 \mu \mathrm{g})$, Amxacillin-Clavulanic acid $(20 \backslash 10 \mu \mathrm{g})$, Ceftazidim $(5 \mu \mathrm{g})$, Cefuroxim $(30 \mu \mathrm{g})$,

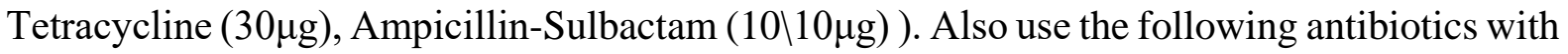
Acinetobacter spp (Ceftazidim $(5 \mu \mathrm{g})$, Impinem $(10 \mu \mathrm{g})$, Tetracycline $(30 \mu \mathrm{g})$, Gentamycin

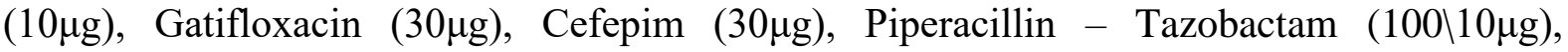
Ciprofloxacin $(30 \mu \mathrm{g})$, Amikacin $(30 \mu \mathrm{g})$, Piperacillin - Tazobactam $(100 \backslash 10 \mu \mathrm{g})$, Levofloxacin $(30 \mu \mathrm{g})$ ). Where use the following antibiotics with Pseudomonas aeruginosa (Impinem $(10 \mu \mathrm{g})$, Gentamycin $(10 \mu \mathrm{g})$, Gatifloxacin $(30 \mu \mathrm{g})$, Cefepim $(30 \mu \mathrm{g})$, Piperacillin - Tazobactam $(100 \backslash 10 \mu \mathrm{g})$, Ofloxacin $(5 \mu \mathrm{g})$, Ciprofloxacin $(30 \mu \mathrm{g})$, Amikacin $(30 \mu \mathrm{g})$, Levofloxacin $(30 \mu \mathrm{g}))$ Where use with Staphylococcus spp ( Pencillin $(10 \mu \mathrm{g})$, Tetracyclin $(30 \mu \mathrm{g})$, Erythromycin $(15 \mu \mathrm{g})$, Clindamycin $(2 \mu \mathrm{g})$, Linezolid $(30 \mu \mathrm{g})$, Nitrofurantoin $(300 \mu \mathrm{g})$, Ciprofloxacin $(30 \mu \mathrm{g})$, Levofloxacin $(30 \mu \mathrm{g})$, Gentamycin $(10 \mu \mathrm{g})$, Ofloxacin $(5 \mu \mathrm{g})$, Gatifloxacin $(30 \mu \mathrm{g})$, Novobiocin $(30 \mu \mathrm{g}))$. Where use the following antibiotics with Enterococcus spp (Levofloxacin $(30 \mu \mathrm{g})$, Tetracyclin $(30 \mu \mathrm{g})$, Fosfomycin $(30 \mu \mathrm{g})$, Gentamycin $(30 \mu \mathrm{g})$, Streptomycin $(10 \mu \mathrm{g})$, Ethromycin $(15 \mu \mathrm{g})$, Gatifloxacin $(30 \mu \mathrm{g})$, Vancomycin $(30 \mu \mathrm{g})$, Pencillin (10 $\mu \mathrm{g})$, Ampicillin $(10 \mu \mathrm{g})$, Linezolid $(30 \mu \mathrm{g})$, Nitrofurantoin $(300 \mu \mathrm{g})$, Ciprofloxacin $(30 \mu \mathrm{g}))$. The choice of the antibiotics was made according to the CLSI guideline of 2019. The clear zone was estimated by millimeter, and the zones were compared with the CLSI standards for interpretation (CLSI, 2013). The reporting was done by indicating Resistant, Intermediate, or Sensitive for all strains.

\section{Results and Discussion}

\subsection{Isolation and Identification of urinary tract bacteria.}

MacConkey agar, CLED agar, Nutrient agar, CTIA media, Mannitol salt agar, and Bile aesculin agar are the media's main roles in isolating and identifying bacteria. Identification 
according to the type of gram stain, either Gram-positive bacteria or Gram-negative bacteria. Both of them have their identification. Gram-positive bacteria include (Catalase and TubeCoagulase test) and make sure to VITEK 2. Gram-negative bacteria include identifying by CTIA tube, which contains five biochemical methods (Glucose and Cellobiose fermentation, $\mathrm{H}_{2} \mathrm{~S}$ production, Tryptophan deaminase activity, and finally indole production) and makes sure to VITEK 2. The bacteria causing urinary tract infection were isolated from 126 patients, while 99 patients were not isolated from them any pathogenic bacteria. In Gram-negative bacteria, the most common bacteria were E. coli (55.5\%), followed by Klebsiella spp (23.0\%), Proteus spp (7.14\%), Pseudomonas spp (6.34\%), and Acinetobacter spp (3.96\%). In the Gram-positive bacteria Staphylococcus spp (2.38\%) and Enterococcus spp (1.58\%) were found. Other report showed the common bacteria were Escherichia coli (77\%), Klebsiella pneumoniae (14.6\%), Proteus mirabilis (4.2\%) Proteus penneri (2.1\%), Enterococcus faecium (2.1\%)[29]. The number and type of bacteria from the infected samples after identification. The definition is later mentioned in detail as follows in Table 1. This is consistent with shows of other studies in which E. coli was the most common reported isolate from patients with UTIs [30], Followed by Klebsiella spp., Proteus spp. and Psedomonas spp. in Gram-negative isolates, whereas in gram-positive isolates maximum were Staphylococcus spp. and Enterococcus spp. Similar shows were reported by several previous international [31,32]. There are several factors in Enterobacteriaceae responsible for the attachment to the urothelium: colonize gram-negative isolates in urogenital mucosa with adhesion, fimbriae, pilli, and P1-blood group phenotype receptors $[33,34]$.

Table 1. Number and type of bacteria from infected samples.

\begin{tabular}{lcc}
\multicolumn{1}{c}{ Strain } & Number & Ratio (\%) \\
Escherichia coli & 70 & 55.5 \\
Klebsiella spp & 29 & 23.0 \\
Acinetobacter spp & 5 & 3.96 \\
Pseudomonas spp & 8 & 6.34 \\
Proteus spp & 9 & 7.14 \\
Enterococcus spp & 2 & 1.58 \\
Staphylococcus spp & 3 & 2.38
\end{tabular}

Seven of these isolates were selected, representing the most resistant to antibiotics and identification by VITEK2 in Tables $2 \& 3$.

Table 2. Identification of Gram-negative bacteria by VITEK2.

\begin{tabular}{|c|c|c|c|c|c|}
\hline Character (Test) & $\begin{array}{c}E . \\
\text { coli }\end{array}$ & $\begin{array}{c}\text { K. } \\
\text { pneumoniae }\end{array}$ & $\begin{array}{c}A . \\
\text { baumannii }\end{array}$ & $\begin{array}{c}P . \\
\text { aeruginosa }\end{array}$ & $\begin{array}{c}\text { Proteus } \\
\text { mirabilis }\end{array}$ \\
\hline Gram stain & - ve & - ve & - ve & - ve & - ve \\
\hline Cell shape & rod & Rod & Cocco bacilli & bacilli & bacilli \\
\hline Ala-Phe-Pro-ARYLAMIDASE & - ve & - ve & $-\mathrm{ve}$ & $+\mathrm{ve}$ & - ve \\
\hline ADONITOL & - ve & $+\mathrm{ve}$ & - ve & $-\mathrm{ve}$ & $-\mathrm{ve}$ \\
\hline L-Pyrrolydonyl-ARYLAMIDASE & $-\mathrm{ve}$ & $+\mathrm{ve}$ & - ve & - ve & - ve \\
\hline L-ARABITOL & - ve & - ve & - ve & - ve & $-\mathrm{ve}$ \\
\hline D-CELLOBIOSE & - ve & $+\mathrm{ve}$ & $+\mathrm{ve}$ & $-\mathrm{ve}$ & - ve \\
\hline BETA-GALACTOSIDASE & $+\mathrm{ve}$ & $+\mathrm{ve}$ & $-\mathrm{ve}$ & - ve & - ve \\
\hline H2S PRODUCTION & - ve & - ve & - ve & - ve & $+\mathrm{ve}$ \\
\hline BETA-N-ACETYL-GLUCOSAMINIDASE & - ve & - ve & - ve & - ve & - ve \\
\hline Glutamyl Arylamidase pNA & - ve & - ve & - ve & - ve & - ve \\
\hline D-GLUCOSE & $+\mathrm{ve}$ & $+\mathrm{ve}$ & $+\mathrm{ve}$ & $+\mathrm{ve}$ & + ve \\
\hline GAMMA-GLUTAMYL-TRANSFERASE & - ve & $+\mathrm{ve}$ & - ve & $+\mathrm{ve}$ & $+\mathrm{ve}$ \\
\hline FERMENTATION $\backslash$ GLUCOSE & $+\mathrm{ve}$ & $+\mathrm{ve}$ & - ve & - ve & - ve \\
\hline BETA-GLUCOSIDASE & - ve & $+\mathrm{ve}$ & $-\mathrm{ve}$ & $-\mathrm{ve}$ & $-\mathrm{ve}$ \\
\hline D-MALTOSE & $+\mathrm{ve}$ & $+\mathrm{ve}$ & - ve & - ve & - ve \\
\hline D-MANNITOL & $+\mathrm{ve}$ & $+\mathrm{ve}$ & - ve & - ve & $-\mathrm{ve}$ \\
\hline D-MANNOSE & $+\mathrm{ve}$ & $+\mathrm{ve}$ & $+\mathrm{ve}$ & $+\mathrm{ve}$ & - ve \\
\hline BETA-XYLOSIDASE & $-\mathrm{ve}$ & $+\mathrm{ve}$ & $-\mathrm{ve}$ & - ve & - ve \\
\hline \multicolumn{5}{|l|}{ https://nanobioletters.com/ } & 2824 \\
\hline
\end{tabular}


https://doi.org/10.33263/LIANBS104.28202830

\begin{tabular}{|c|c|c|c|c|c|}
\hline Character (Test) & $\begin{array}{c}E . \\
\text { coli }\end{array}$ & $\begin{array}{c}\text { K. } \\
\text { pneumoniae }\end{array}$ & $\begin{array}{c}\text { A. } \\
\text { baumannii }\end{array}$ & $\begin{array}{c}P . \\
\text { aeruginosa }\end{array}$ & $\begin{array}{l}\text { Proteus } \\
\text { mirabilis }\end{array}$ \\
\hline BETA-Alanine arylamidase pNA & - ve & $-\mathrm{ve}$ & $-\mathrm{ve}$ & + ve & - ve \\
\hline L-Proline ARYLAMIDASE & $+\mathrm{ve}$ & $+\mathrm{ve}$ & $-\mathrm{ve}$ & + ve & $-\mathrm{ve}$ \\
\hline LIPASE & - ve & - ve & - ve & + ve & - ve \\
\hline PALATINOSE & - ve & + ve & $-\mathrm{ve}$ & $-\mathrm{ve}$ & - ve \\
\hline Tyrosine ARYLAMIDASE & + ve & + ve & - ve & + ve & + ve \\
\hline UREASE & - ve & $-\mathrm{ve}$ & $-\mathrm{ve}$ & + ve & + ve \\
\hline D-SORBITOL & $+\mathrm{ve}$ & + ve & $-\mathrm{ve}$ & $-\mathrm{ve}$ & $-\mathrm{ve}$ \\
\hline SACCHAROSEISUCROSE & $+\mathrm{ve}$ & $+\mathrm{ve}$ & $-\mathrm{ve}$ & $-\mathrm{ve}$ & - ve \\
\hline D-TAGATOSE & + ve & - ve & - ve & - ve & - ve \\
\hline D-TREHALOSE & + ve & + ve & - ve & $-\mathrm{ve}$ & - ve \\
\hline CITRATE(SODIUM) & - ve & - ve & + ve & + ve & - ve \\
\hline MALINATE & - ve & + ve & + ve & + ve & - ve \\
\hline 5-KETO-D-GLUCONATE & - ve & $-\mathrm{ve}$ & - ve & $-\mathrm{ve}$ & - ve \\
\hline L-LACTATE alkalinisation & + ve & + ve & + ve & + ve & $+\mathrm{ve}$ \\
\hline ALPHA-GLUCOSIDASE & - ve & - ve & - ve & - ve & - ve \\
\hline SUCCINATE alkalinisation & + ve & + ve & + ve & + ve & + ve \\
\hline $\begin{array}{l}\text { BETA-N-ACETYL- } \\
\text { GALACTOSAMINIDASE }\end{array}$ & - ve & - ve & - ve & - ve & - ve \\
\hline ALPHA-GALACTOSIDASE & + ve & $+\mathrm{ve}$ & - ve & - ve & $-\mathrm{ve}$ \\
\hline PHOSPHATASE & - ve & + ve & - ve & $-\mathrm{ve}$ & + ve \\
\hline Glycine ARYLAMIDASE & - ve & - ve & - ve & - ve & - ve \\
\hline ORNITHINE DECARBOXYLASE & + ve & - ve & - ve & - ve & + ve \\
\hline LYSINE DECARBOXYLASE & + ve & + ve & - ve & $-\mathrm{ve}$ & - ve \\
\hline L-HISTIDINE assimilation & - ve & $-\mathrm{ve}$ & + ve & $-\mathrm{ve}$ & $-\mathrm{ve}$ \\
\hline COUMARATE & + ve & - ve & + ve & + ve & + ve \\
\hline BETA-GLUCORONIDASE & - ve & $-\mathrm{ve}$ & - ve & $-\mathrm{ve}$ & - ve \\
\hline O129 RESISTANCE(comp.vibrio.) & $+\mathrm{ve}$ & + ve & + ve & + ve & + ve \\
\hline Glu-Gly-Arg-ARYLAMIDASE & - ve & - ve & - ve & + ve & - ve \\
\hline L-MALATE assimilation & - ve & - ve & + ve & + ve & + ve \\
\hline ELLMAN & $-\mathrm{ve}$ & $-\mathrm{ve}$ & - ve & $-\mathrm{ve}$ & - ve \\
\hline L-LACTATE assimilation & - ve & - ve & $+\mathrm{ve}$ & + ve & - ve \\
\hline
\end{tabular}

Tables 3. Identification of Gram-positive bacteria by VITEK2.

Character (Test)

\begin{tabular}{|c|c|c|}
\hline Character (Test) & Staphylococcus arlettae & Enterococcus faecalis \\
\hline Gram stain & $+\mathrm{ve}$ & $+\mathrm{ve}$ \\
\hline Cell shape & Cocci & Cocci \\
\hline Catalase & $+\mathrm{ve}$ & - ve \\
\hline D-Amygdalin & - ve & $+\mathrm{ve}$ \\
\hline Ala-phe-pro- Arylamidase & $-\mathrm{ve}$ & $-\mathrm{ve}$ \\
\hline Leucine Arylamidase & - ve & - ve \\
\hline Alanine Arlamidase & - ve & - ve \\
\hline D-Ribose & $+\mathrm{ve}$ & $+\mathrm{ve}$ \\
\hline Novobiocin Resistance & $+\mathrm{ve}$ & $+\mathrm{ve}$ \\
\hline D- Raffinose & $+\mathrm{ve}$ & - ve \\
\hline Optochin resistance & + ve & + ve \\
\hline Phosphatidylinositol Phospholipase C & - ve & - ve \\
\hline Cyclodextrin & - ve & $+\mathrm{ve}$ \\
\hline L-Prolin Arylamidase & - ve & - ve \\
\hline Tyrosine Arylamidase & - ve & $+\mathrm{ve}$ \\
\hline L-Lactate Alkalinization & - ve & - ve \\
\hline Growth in $6.5 \% \mathrm{NaCl}$ & $+\mathrm{ve}$ & $+\mathrm{ve}$ \\
\hline $\mathrm{O} / 129$ resistance & + ve & - ve \\
\hline D-Xylose & - ve & - ve \\
\hline L- Aspartate Arylamidase & - ve & + ve \\
\hline (Beta- Glucuronidase) & - ve & - ve \\
\hline D-Sorbitol & - ve & $+\mathrm{ve}$ \\
\hline Lactose & $+\mathrm{ve}$ & + ve \\
\hline D-manitol & $+\mathrm{ve}$ & $+\mathrm{ve}$ \\
\hline Salicin & - ve & + ve \\
\hline Argnine Dihydrolase & - ve & + ve \\
\hline Beta galactopyranosidase & - ve & - ve \\
\hline Alpha- galactocidase & - ve & - ve \\
\hline N- Acetyle-D- Glucosamine & $-\mathrm{ve}$ & + ve \\
\hline D-Mannose & $-\mathrm{ve}$ & + ve \\
\hline Sucrose & - ve & + ve \\
\hline Alpha-Mannosidase & + ve & - ve \\
\hline
\end{tabular}




\begin{tabular}{l|c|c} 
Character (Test) & Staphylococcus arlettae & Enterococcus faecalis \\
\hline Beta-galactosidase & $-\mathrm{ve}$ & $-\mathrm{ve}$ \\
\hline L-Pyrrolidonyl-Arylamidase & $-\mathrm{ve}$ & $+\mathrm{ve}$ \\
\hline Polymixin B resistance & $-\mathrm{ve}$ & $+\mathrm{ve}$ \\
\hline D-maltose & $-\mathrm{ve}$ & $+\mathrm{ve}$ \\
\hline Methyl-B-D-Glucopyranoside & $+\mathrm{ve}$ & $+\mathrm{ve}$ \\
\hline D-Trehalose & $-\mathrm{ve}$ & $+\mathrm{ve}$ \\
\hline Alpha-glucosidase & $+\mathrm{ve}$ & $+\mathrm{ve}$ \\
\hline Beta Glucuronidase & $-\mathrm{ve}$ & $-\mathrm{ve}$ \\
\hline Phosphatase & $+\mathrm{ve}$ & $-\mathrm{ve}$ \\
\hline D-Galactose & $-\mathrm{ve}$ & $+\mathrm{ve}$ \\
\hline Bacitracine Resistance & $+\mathrm{ve}$ & $+\mathrm{ve}$ \\
\hline Pullulan & $-\mathrm{ve}$ & $-\mathrm{ve}$ \\
\hline Arginine Dihydrolase2 & $-\mathrm{ve}$ & $+\mathrm{ve}$
\end{tabular}

\subsection{Antimicrobial susceptibility for Gram-negative bacteria.}

Antimicrobial susceptibility for Gram-negative bacteria in Table 4. The results showed that $E$. coli bacteria are more resistant to antibiotics such as Cefuroxime 100\%, Ceftazedim 97.2\%, Tetracyclin 97.2\%, Ofloxacin 96.98\%, Amoxicillin-clavulanic acid 95.78\%, Cefoxitine $90.15 \%$, Ampicillin 87.4\%, Cefepime 85.92\%, Piperacillin-Tazobactam 83.1\%, Cefotaxim 78.9\%, Naldxic acid 76.1\%, Ciprofloxacin 69.1\%, Gatifloxacin 56.4\% and Levofloxacin $56.34 \%$. While it was sensitive to the antibiotics represented in Amikacin 100\%, Impinem 97.1\%, Fosfomycin 83.09\%, Nitrofurantoin 64.7\%, and streptomycin 60.5\%. Klebsiella pneumoniae showed more resistance to antibiotics such as Ceftazedim $100 \%$, Tetracyclin 100\%, Cefuroxim 100\%, Ampicillin-Sulbactam 100\%, Amoxicillin-clavulanic acid 96.56, Ampicillin 93.11\%, Cefoxitin 89.66\%, Cefepim 89.66\%, Cefotaxime 86.03\%, Nitrofurantoin 75.9\%, Fosfomycin 75.87\%, Piperacillin-Tazobactam $68.97 \%$ and Ciprofloxacin $62.07 \%$. While it was sensitive to the antibiotics represented in Amikacin 93.1\%, Levofloxacin 86.2\%, Impinem 79.3\%, Ofloxacin $75.86 \%$, Gatifloxacin $72.4 \%$, and Nalidixic acid $68.96 \%$. Proteus mirabilis showed more resistant for Ceftazedim $100 \%$, Tetracyclin 100\%, Cefuroxim 100\%, Cefoxitin 100\%, Cefepime 100\%, Cefotaxime 100\%, Ampicillin-Sulbactam 100\%, Amoxicillin-clavulanic acid 88.9, Ampicillin 88.9\%, Nalidixic acid $77.8 \%$, Fosfomycin $77.8 \%$, Streptomycin 66.7\%, Ciprofloxacin 66.7\%, Levofloxacin 60\%, Ofloxacin $55.6 \%$ and Nitrofurantoin 55.6\%. While it was sensitive to the antibiotics represented in Amikacin 77.7\%, Impinem 66.6\%, Gatifloxacin 55.5\%, Piperacillin-Tazobactam 55.5\%, and Levofloxacin $55.5 \%$. Acinetobacter baumannii showed more resistance for Ceftazidim 100\%, Tetracyclin 80\%, Piperacillin-Tazobactam 80\%, Ciprofloxacin 80\%, Ampicillin-Tazobactam 80\%, and Gatifloxacin $60 \%$. In comparison, it was sensitive for Amikacin 100\% and Impinem $60 \%$. The results showed that Pseudomonas aeruginosa bacteria are more resistant to antibiotics such as Cefepime 100\%, Ciprofloxacin $62.5 \%$, and Piperacillin-Tazobactam 50\%. While it was sensitive to the antibiotics represented in Impinem 100\%, Amikaci 100\%, Levofloxacin $75.0 \%$, Gatifloxacin $62.5 \%$, and Ofloxacin $62.5 \%$. A comparative with other study demonstrates high fluoroquinolone and aminoglycoside resistance among most Gram-negative species; and carbapenem resistance among Pseudomonas spp and Acinetobacter spp [35], imipenem, azithromycin, and cephalexin demonstrated sensitivity pattern for most of the isolated organisms showed 50\% and/or higher [36], E. coli was most susceptible to meropenem (91.89\%) closely followed by imipenem $(91.69 \%)$. E. coli had the least susceptibility to cefuroxime (18.18\%) and ciprofloxacin $(18.97 \%)$. Klebsiella spp was most susceptible to imipenem $(75.89 \%)$, closely followed by meropenem $(75.49 \%)$. Klebsiella spp. was least susceptible to ampicillin (2.37\%). Pseudomonas spp. was most susceptible to polymyxin B 
(92.19\%) followed by aztreonam (68.29\%). Pseudomonas spp. was least susceptible to ciprofloxacin (25.85\%) [37]. Another study explains the resistance for Proteus mirabilis, the highest prevalence of resistance was cotrimoxazole (>30\%), while quinolone and fosfomycin resistance remained below $20 \%$ throughout the study period. However, for quinolones, resistance increased from $11.8 \%$ in 2009 to $17.6 \%$ in 2016 [38]. It is noticeable from the results that pathogenic bacteria (E. coli, Klebsiella pneumoniae, Proteus mirabilis, Acinetobacter baumannii, and Pseudomonas aeruginosa) have developed in their resistance to antibiotics over the previous years.

Table 4. Antimicrobial susceptibility rate of isolated Gram-negative bacteria uropathogens, N=121.

\begin{tabular}{|c|c|c|c|c|c|}
\hline Antibiotics & $\begin{array}{c}\text { E. coli } \\
\mathrm{N}=70(55.5 \%)\end{array}$ & $\begin{array}{c}\text { K. pneumoniae } \\
\mathrm{N}=29(23 \%)\end{array}$ & $\begin{array}{c}\text { Proteus } \\
\text { mirabilis } \\
\mathrm{N}=9(7.14 \%)\end{array}$ & $\begin{array}{c}\text { Acinetobacter } \\
\text { baumannii } \\
\mathrm{N}=5(3.96 \%)\end{array}$ & $\begin{array}{c}\text { Pseudomonas } \\
\text { aeruginosa } \\
\mathrm{N}=8(6.34 \%)\end{array}$ \\
\hline Nitrofurantoin & $\mathrm{N}=46(64.7 \%)$ & $\mathrm{N}=7(24.1 \%)$ & $\mathrm{N}=4(44.4 \%)$ & $\mathrm{N}=0$ & $\mathrm{~N}=0$ \\
\hline Ceftazedime & $\mathrm{N}=2(2.8 \%)$ & $\mathrm{N}=0$ & $\mathrm{~N}=0$ & $\mathrm{~N}=0$ & $\mathrm{~N}=0$ \\
\hline Impinem & $\mathrm{N}=69(97.1 \%)$ & $\mathrm{N}=23(79.3 \%)$ & $\mathrm{N}=6(66.6 \%)$ & $\mathrm{N}=3(60 \%)$ & $\mathrm{N}=8(100 \%)$ \\
\hline Tetracycline & $\mathrm{N}=2(2.8 \%)$ & $\mathrm{N}=0$ & $\mathrm{~N}=0$ & $\mathrm{~N}=1(20 \%)$ & $\mathrm{N}=0$ \\
\hline Streptomycin & $\mathrm{N}=43(60.5 \%)$ & $\mathrm{N}=19(65.5 \%)$ & $\mathrm{N}=3(33.3 \%)$ & $\mathrm{N}=0$ & $\mathrm{~N}=0$ \\
\hline Cefuroxime & $\mathrm{N}=0$ & $\mathrm{~N}=0$ & $\mathrm{~N}=0$ & $\mathrm{~N}=0$ & $\mathrm{~N}=0$ \\
\hline Gatifloxa-cin & $\mathrm{N}=31(43.6 \%)$ & $\mathrm{N}=21(72.4 \%)$ & $\mathrm{N}=5(55.5 \%)$ & $\mathrm{N}=2(40.0 \%)$ & $\mathrm{N}=5(62.5 \%)$ \\
\hline Ampicillin & $\mathrm{N}=9(12.6 \%)$ & $\mathrm{N}=2(6.89 \%)$ & $\mathrm{N}=1(11.1 \%)$ & $\mathrm{N}=0$ & $\mathrm{~N}=0$ \\
\hline Cefoxitin & $\mathrm{N}=7(9.85 \%)$ & $\mathrm{N}=3(10.34 \%)$ & $\mathrm{N}=0$ & $\mathrm{~N}=0$ & $\mathrm{~N}=0$ \\
\hline Nalidixic acid & $\mathrm{N}=17(23.9 \%)$ & $\mathrm{N}=20(68.96 \%)$ & $\mathrm{N}=2(22.2 \%)$ & $\mathrm{N}=0$ & $\mathrm{~N}=0$ \\
\hline Cefepime & $\mathrm{N}=10(14.08 \%)$ & $\mathrm{N}=3(10.34 \%)$ & $\mathrm{N}=0$ & $\mathrm{~N}=0$ & $\mathrm{~N}=0$ \\
\hline Fosfomycin & $\mathrm{N}=59(83.09 \%)$ & $\mathrm{N}=7(24.13 \%)$ & $\mathrm{N}=2(22.2 \%)$ & $\mathrm{N}=0$ & $\mathrm{~N}=0$ \\
\hline Piperacillin - Tazobactam & $\mathrm{N}=12(16.9 \%)$ & $\mathrm{N}=9(31.03 \%)$ & $\mathrm{N}=5(55.5 \%)$ & $\mathrm{N}=1(20 / 0 \%)$ & $\mathrm{N}=4(50 \%)$ \\
\hline Ofloxacin & $\mathrm{N}=27(38.02 \%)$ & $\mathrm{N}=22(75.86 \%)$ & $\mathrm{N}=4(44.4 \%)$ & $\mathrm{N}=0$ & $\mathrm{~N}=5(62.5 \%)$ \\
\hline Cefotaxim & $\mathrm{N}=15(21.1 \%)$ & $\mathrm{N}=4(13.97 \%)$ & $\mathrm{N}=0$ & $\mathrm{~N}=0$ & $\mathrm{~N}=0$ \\
\hline Ciprofloxacin & $\mathrm{N}=22(30.9 \%)$ & $\mathrm{N}=11(37.93 \%)$ & $\mathrm{N}=3(33.3 \%)$ & $\mathrm{N}=1(20.0 \%)$ & $\mathrm{N}=3(37.5 \%)$ \\
\hline Amikacin & $\mathrm{N}=70(100 \%)$ & $\mathrm{N}=27(93.1 \%)$ & $\mathrm{N}=7(77.7 \%)$ & $\mathrm{N}=5(100 \%)$ & $\mathrm{N}=8(100 \%)$ \\
\hline Ampicillin-Sulbactam & $\mathrm{N}=3(4.22 \%)$ & $\mathrm{N}=0$ & $\mathrm{~N}=0$ & $\mathrm{~N}=1(20.0 \%)$ & $\mathrm{N}=0$ \\
\hline Levofloxacin & $\mathrm{N}=31(43.66 \%)$ & $\mathrm{N}=25(86.2 \%)$ & $\mathrm{N}=5(55.5 \%)$ & $\mathrm{N}=2(40.0 \%)$ & $\mathrm{N}=6(75 \%)$ \\
\hline Amoxicillin-Clavulanic acid & $\mathrm{N}=3(4.22 \%)$ & $\mathrm{N}=1(3.44 \%)$ & $\mathrm{N}=1(11.1 \%)$ & $\mathrm{N}=0$ & $\mathrm{~N}=0$ \\
\hline
\end{tabular}

\subsection{Antimicrobial susceptibility for Gram-positive bacteria.}

Antimicrobial susceptibility for Gram-positive bacteria is presented in Table 5.

Table 5. Antimicrobial susceptibility rate of isolated Gram-positive bacteria uropathogens, N=5.

\begin{tabular}{l|c|c} 
Gram-positive bacteria & $\begin{array}{c}\text { Staphylococcus arlettae } \\
\mathbf{N}=\mathbf{3}(\mathbf{2 . 3 8} \%)\end{array}$ & $\begin{array}{c}\text { Enterococcus faecalis } \\
\mathbf{N}=\mathbf{2}(\mathbf{1 . 5 8} \%)\end{array}$ \\
\hline Penicillin & $\mathrm{N}=0$ & $\mathrm{~N}=0$ \\
\hline Tetracyclin & $\mathrm{N}=0$ & $\mathrm{~N}=2(100 \%)$ \\
\hline Erythromycin & $\mathrm{N}=0$ & $\mathrm{~N}=2(100 \%)$ \\
\hline Clindamycin & $\mathrm{N}=0$ & $\mathrm{~N}=0$ \\
\hline Linezolid & $\mathrm{N}=0$ & $\mathrm{~N}=2(100 \%)$ \\
\hline Nitrofurantoin & $\mathrm{N}=0$ & $\mathrm{~N}=1(50 \%)$ \\
\hline Ciprofloxacin & $\mathrm{N}=1(33.3 \%)$ & $\mathrm{N}=2(100 \%)$ \\
\hline Levofloxacin & $\mathrm{N}=2(66.6 \%)$ & $\mathrm{N}=2(100 \%)$ \\
\hline Ofloxacin & $\mathrm{N}=2(66.6 \%)$ & $\mathrm{N}=0$ \\
\hline Gatifloxacin & $\mathrm{N}=0$ & $\mathrm{~N}=2(100 \%)$ \\
\hline Novobiocin & $\mathrm{N}=0$ & $\mathrm{~N}=0$ \\
\hline Fosfomycin & $\mathrm{N}=0$ & $\mathrm{~N}=0$ \\
\hline Streptomycin & $\mathrm{N}=0$ & $\mathrm{~N}=2(100 \%)$ \\
\hline Vancomycin & $\mathrm{N}=0$ & $\mathrm{~N}=1(50 \%)$ \\
\hline Ampicillin & $\mathrm{N}=0$ & $\mathrm{~N}=0$
\end{tabular}


The results showed that Staphylococcus arlettae bacteria are more resistant to antibiotics such as Penicillin 100\%, Tetracyclin 100\%, Erythromycin 100\%, Clindamycin $100 \%$, Linezolid 100\%, Nitrofurantoin 100\%, Gatifloxacin 100\%, Novobiocin $100 \%$, and Ciprofloxacin $66.7 \%$. While it was sensitive to the antibiotics represented in levofloxacin 66.6\% and Ofloxacin 66.6\%. Enterococcus faecalis showed more resistant for Penicillin 100\% \& Ampicillin 100\% \& Fosfomycin 100\% \& Nitrofurantoin 50\% and Vancomycin 50\%. While it was sensitive to the antibiotics represented in Tetracycline, Erythromycin, Linezolid, Ciprofloxacin, Levofloxacin, Gatifloxacin, and Streptomycin, each of them represent 50\%, and others represent less than 50\%. Another study demonstrated that Enterococcus spp. and Staphylococcus spp. were most susceptible to linezolid at $96.55 \%$ and $100 \%$, respectively, followed by vancomycin at $87.93 \%$ and $100 \%$, respectively [37].

\section{Conclusions}

The problem of urinary tract infection and the identification of harmful bacterial causes are of great importance. Therefore, the study was based on clarifying the positive and Gramnegative bacterial isolates that affect the urinary tract and identifying these isolates' antibiotic resistance profiles. As these isolates were defined by morphological, biochemical, and VITEK 2 methods, the results showed that the bacteria were represented in E. coli (55.5\%), Klebsiella pneumoniae (23.0\%), Proteus mirabilis (7.14\%), Pseudomonas aeruginosa (6.34\%), Acinetobacter baumannii ( 3.96\%), Staphylococcus arlettae (2.38\%), Enterococcus faecalis $(1.58 \%)$ and showed resistance to most antibiotics. Therefore, it is necessary to shed light on this problem and the extent of microbes' development that affect the urinary tract and their resistance to most antibiotics.

\section{Funding}

This research received no external funding.

\section{Acknowledgments}

The authors express their sincere thanks to the Faculty of Science (Boyes), Al-Azhar University, Cairo, Egypt, for providing the necessary research facilities.

\section{Conflicts of Interest}

The authors declare no conflict of interest.

\section{References}

1. Klein, R.D.; Hultgren, S.J. Urinary tract infections: microbial pathogenesis, host-pathogen interactions and new treatment strategies. Nature Reviews Microbiology 2020, 18, 211-226, https://doi.org/10.1038/s41579020-0324-0.

2. Dospinescu, V.-M.; Tiele, A.; Covington, J.A. Sniffing Out Urinary Tract Infection-Diagnosis Based on Volatile Organic Compounds and Smell Profile. Biosensors 2020, 10, 83, https://doi.org/10.3390/bios10080083.

3. Folliero, V.; Caputo, P.; Della Rocca, M.T.; Chianese, A.; Galdiero, M.; Iovene, M.R.; Hay, C.; Franci, G.; Galdiero, M. Prevalence and antimicrobial susceptibility patterns of bacterial pathogens in urinary tract infections in University Hospital of Campania "Luigi Vanvitelli" between 2017 and 2018. Antibiotics 2020, 9, 215, https://doi.org/10.3390/antibiotics9050215. 
4. Flores-Mireles, A.L.; Walker, J.N.; Caparon, M.; Hultgren, S.J. Urinary tract infections: epidemiology, mechanisms of infection and treatment options. Nature reviews microbiology 2015, 13, 269-284, https://doi.org/10.1038/nrmicro3432.

5. Storme, O.; Tiran Saucedo, J.; Garcia-Mora, A.; Dehesa-Dávila, M.; Naber, K.G. Risk factors and predisposing conditions for urinary tract infection. Therapeutic advances in urology 2019, 11, 1756287218814382, https://doi.org/10.1177/1756287218814382.

6. Schwartz, K.L.; Morris, S.K. Travel and the spread of drug-resistant bacteria. Current infectious disease reports 2018, 20, 1-10, https://doi.org/10.1007/s11908-018-0634-9.

7. Rexhepi, M.; Besimi, F.; Rufati, N.; Alili, A.; Bajrami, S.; Ismaili, H. Hospital-based study of maternal, perinatal and neonatal outcomes in adolescent pregnancy compared to adult women pregnancy. Open access Macedonian journal of medical sciences 2019, 7, 760, https://doi.org/10.3889/oamjms.2019.210.

8. Ailes, E.C.; Summers, A.D.; Tran, E.L.; Gilboa, S.M.; Arnold, K.E.; Meaney-Delman, D.; Reefhuis, J. Antibiotics dispensed to privately insured pregnant women with urinary tract infections-United States, 2014. Morbidity and Mortality Weekly Report 2018, 67, 18, https://doi.org/10.15585/mmwr.mm6701a4.

9. El-Sahrigy, S.A.; Shouman, M.G.; Ibrahim, H.M.; Rahman, A.M.A.; Habib, S.A.; Khattab, A.A.; Gomaa, H.E.; Helmy, N.A. Prevalence and antimicrobial susceptibility of hospital acquired infections in two pediatric intensive care units in Egypt. Open access Macedonian journal of medical sciences 2019, 7, 1744, https://doi.org/10.3889/oamjms.2019.485.

10. Hvidberg, H.; Struve, C.; Krogfelt, K.A.; Christensen, N.; Rasmussen, S.N.; Frimodt-Møller, N. Development of a long-term ascending urinary tract infection mouse model for antibiotic treatment studies. Antimicrobial agents and chemotherapy 2000, 44, 156-163, http://pascalfrancis.inist.fr/vibad/index.php?action=getRecordDetail\&idt=1259055.

11. Öztürk, R.; Murt, A. Epidemiology of urological infections: A global burden. World journal of urology 2020, 10.1007/s00345-019-03071-4, 1-11, https://doi.org/10.1007/s00345-019-03071-4.

12. Kumar, A.; Jhadwal, N.; Lal, M. Antibacterial activity of some medicinal plants used against UTI causing pathogens. 2012, https://www.sid.ir/en/Journal/ViewPaper.aspx?ID=498102.

13. Ahmed, S.S.; Shariq, A.; Alsalloom, A.A.; Babikir, I.H.; Alhomoud, B.N. Uropathogens and their antimicrobial resistance patterns: Relationship with urinary tract infections. International journal of health sciences 2019, 13, 48, https://www.ncbi.nlm.nih.gov/pmc/articles/PMC6436442/.

14. Chu, C.M.; Lowder, J.L. Diagnosis and treatment of urinary tract infections across age groups. American journal of obstetrics and gynecology 2018, 219, 40-51, https://doi.org/10.1016/j.ajog.2017.12.231.

15. Desouky, S.E.; El-Gamal, M.S.; Mohammed, A.F.; Abu-Elghait, M.A. Determination of some virulence factors in Staphylococcus spp. isolated from clinical samples of different Egyptian patients. World Appl Sci $J$ 2014, 32, 731-740, https://www.idosi.org/wasj/wasj32(4)14/30.pdf.

16. Mazzariol, A.; Bazaj, A.; Cornaglia, G. Multi-drug-resistant Gram-negative bacteria causing urinary tract infections: a review. Journal of Chemotherapy 2017, 29, 2-9, https://doi.org/10.1080/1120009X.2017.1380395.

17. Yasir, M.; Ajlan, A.M.; Shakil, S.; Jiman-Fatani, A.A.; Almasaudi, S.B.; Farman, M.; Baazeem, Z.M.; Baabdullah, R.; Alawi, M.; Al-Abdullah, N. Molecular characterization, antimicrobial resistance and clinicobioinformatics approaches to address the problem of extended-spectrum $\beta$-lactamase-producing Escherichia coli in western Saudi Arabia. Scientific reports 2018, 8, 1-11, https://doi.org/10.1038/s41598-018-33093-8.

18. Oteo, J.; Pérez-Vázquez, M.; Campos, J. Extended-spectrum $\beta$-lactamase producing Escherichia coli: changing epidemiology and clinical impact. Current opinion in infectious diseases 2010, 23, 320-326, https://doi.org/10.1097/qco.0b013e3283398dc1.

19. Rather, I.A.; Kim, B.-C.; Bajpai, V.K.; Park, Y.-H. Self-medication and antibiotic resistance: Crisis, current challenges, and prevention. Saudi journal of biological sciences 2017, 24, 808-812, https://doi.org/10.1016/j.sjbs.2017.01.004.

20. Brumbaugh, A.R.; Mobley, H.L. Preventing urinary tract infection: progress toward an effective Escherichia coli vaccine. Expert review of vaccines 2012, 11, 663-676, https://doi.org/10.1586/erv.12.36.

21. Hassan, D.; Ibrahim, G.; Ibrahim, D. A novel single tube method for biochemical identification of Escherichia coli. Life Science Journal. Recuperado el 2016, 23, https://doi.org/10.7537/marslsj130816.02.

22. Almalki, M.A.; Varghese, R. Prevalence of catheter associated biofilm producing bacteria and their antibiotic sensitivity pattern. Journal of King Saud University-Science 2020, 32, 1427-1433, https://doi.org/10.1016/j.jksus.2019.11.037. 
23. Guerrero, R. Bergey's manuals and the classification of prokaryotes. International microbiology : the official journal of the Spanish Society for Microbiology 2001, 4, 103-109, https://doi.org/10.1007/s101230100021.

24. Kovacs, N. Identification of Pseudomonas pyocyanea by the oxidase reaction. Nature 1956, 178, 703-703, https://doi.org/10.1038/178703a0.

25. Brown, D.F.; Edwards, D.I.; Hawkey, P.M.; Morrison, D.; Ridgway, G.L.; Towner, K.J.; Wren, M.W. Guidelines for the laboratory diagnosis and susceptibility testing of methicillin-resistant Staphylococcus aureus (MRSA). Journal of antimicrobial chemotherapy 2005, 56, 1000-1018, https://doi.org/10.1093/jac/dki372.

26. El-gamal, M.S.; Desouky, S.E.; Hassan, S.E.; Elghait, M.A. Phenotypic and biochemical characterization of specific virulence determinants regulated By Agr quorum sensing system in Staphylococcus aureus. Al Azhar Bull. Sci. 2017.

27. Weinstein, M.P.; Lewis, J.S., 2nd. The Clinical and Laboratory Standards Institute Subcommittee on Antimicrobial Susceptibility Testing: Background, Organization, Functions, and Processes. J Clin Microbiol 2020, 58, e01864-01819, https://doi.org/10.1128/JCM.01864-19.

28. Jorgensen, J.H.; Turnidge, J.D. Susceptibility Test Methods: Dilution and Disk Diffusion Methods. In Manual of Clinical Microbiology, 2015, 1253-1273; https://doi.org/10.1128/9781555817381.ch71..

29. Page, A.-L.; de Rekeneire, N.; Sayadi, S.; Aberrane, S.; Janssens, A.-C.; Rieux, C.; Djibo, A.; Manuguerra, J.-C.; Ducou-le-Pointe, H.; Grais, R.F. Infections in children admitted with complicated severe acute malnutrition in Niger. PloS one 2013, 8, e68699, https://doi.org/10.1371/journal.pone.0068699.

30. Dimitrov, T.; Udo, E.; Emara, M.; Awni, F.; Passadilla, R. Etiology and antibiotic susceptibility patterns of community-acquired urinary tract infections in a Kuwait hospital. Medical principles and Practice 2004, 13, 334-339, https://doi.org/10.1159/000080470.

31. Sharifian, M.; Karimi, A.; Tabatabaei, S.R.; Anvaripour, N. Microbial sensitivity pattern in urinary tract infections in children: a single center experience of 1,177 urine cultures. Japanese journal of infectious diseases 2006, 59, 380, https://pubmed.ncbi.nlm.nih.gov/17186957/.

32. Ahmed, E. Urinary tract bacterial pathogens and their sensitivity pattern. Journal of Rawalpindi Medical College 2014, 18, 263-264, http://journalrmc.com/index.php/JRMC/article/view/357.

33. Das, R.; Chandrashekhar, T.; Joshi, H.; Gurung, M.; Shrestha, N.; Shivananda, P. Frequency and susceptibility profile of pathogens causing urinary tract infections at a tertiary care hospital in western Nepal. Singapore medical journal 2006, 47, 281, https://www.sma.org.sg/smj/4704/4704a5.pdf.

34. Neal, D.E. HOST DEFENSE MECHANISMS IN URINARY TRACT INFECTIONS. Urologic Clinics of North America 1999, 26, 677-686, https://doi.org/10.1016/S0094-0143(05)70210-X.

35. Jones, R.N. Impact of changing pathogens and antimicrobial susceptibility patterns in the treatment of serious infections in hospitalized patients. The American journal of medicine 1996, 100, 3S-12S, https://doi.org/10.1016/S0002-9343(96)00102-7.

36. Akter, T.; Mia, Z.; Shahriar, M. Antibiotic sensitivity of pathogens causing urinary tract infection. Bangladesh Pharmaceutical Journal 2013, 16, 53-58, https://doi.org/10.3329/bpj.v16i1.14491.

37. Patel, H.B.; Soni, S.T.; Bhagyalaxmi, A.; Patel, N.M. Causative agents of urinary tract infections and their antimicrobial susceptibility patterns at a referral center in Western India: An audit to help clinicians prevent antibiotic misuse. Journal of family medicine and primary care 2019, 8, 154, https://doi.org/10.4103/jfmpc.jfmpc_203_18.

38. Zanichelli, V.; Huttner, A.; Harbarth, S.; Kronenberg, A.O.; Huttner, B. Antimicrobial resistance trends in Escherichia coli, Klebsiella pneumoniae and Proteus mirabilis urinary isolates from Switzerland: retrospective analysis of data from a national surveillance network over an 8-year period (2009-2016). Swiss medical weekly 2019, 149, https://doi.org/10.4414/smw.2019.20110. 